\title{
Cognitive remediation group therapy versus mutual aid group therapy: A pilot randomized, controlled trial to determine the feasibility and acceptability of group therapy for people aging with HIV facing cognitive challenges
}

Andrew David Eaton ( $\nabla$ andrew.eaton@utoronto.ca )

University of Toronto https://orcid.org/0000-0003-1331-1222

Shelley L. Craig University of Toronto Factor-Inwentash Faculty of Social Work

Sean B. Rourke

St Michael's Hospital

\section{Teresa Sota}

St Michael's Hospital

John W. McCullagh

Ontario AIDS Network

\section{Barbara A. Fallon}

University of Toronto Factor-Inwentash Faculty of Social Work

Sharon L. Walmsley

Toronto General Research Institute

\section{Research}

Keywords: HIV \& AIDS, group therapy, cognition, aging, pilot randomized controlled trial, Canada

Posted Date: April 9th, 2020

DOI: https://doi.org/10.21203/rs.3.rs-21835/v1

License: (c) (i) This work is licensed under a Creative Commons Attribution 4.0 International License.

Read Full License 


\section{Abstract}

Background: Cognitive impairment is an important comorbidity for people aging with HIV, yet we lack non-medical techniques to address the associated anxiety and stress. Combination psychosocial interventions may have better outcomes than single technique approaches. Mindfulness-Based Stress Reduction (MBSR) and tablet-based Brain Training Activities (BTA) are promising techniques. Using community-based participatory research, our objective was to determine the feasibility and acceptability of group therapy for HIV, aging, and cognition.

Methods: A pilot, parallel design, two-arm RCT recruited from a Toronto neurobehavioural research unit. Eligibility criteria included: diagnoses with mild-to-moderate HIV-associated neurocognitive disorder (HAND), age $\geq 40$ years, HIV-positive for 5+ years, and English fluency. Randomization was 1:1 concealed allocation to Cognitive Remediation Group Therapy (Experimental; combination of BTA and MBSR) or Mutual Aid Group Therapy (Control). Primary outcomes were feasibility, measured by recruitment and completion, and acceptability, determined by a satisfaction questionnaire. The secondary outcome was intervention fidelity, assessed via facilitator session reports. Exploratory outcomes were anxiety, stress, coping, and use of mindfulness and brain training activities. Facilitators and analysts were blinded, however participants were not.

Results: From 1 April 2018 to 19 March 2019, forty eligible participants were contacted, 12 randomized, and 10 completed the study and were analyzed for outcomes. The trial met its a priori feasibility targets with $30 \%$ recruited and $25 \%$ completed. At post-intervention, acceptability was $90 \%$ in the novel and $85 \%$ in the control arm. Assessors confirmed intervention delivery with satisfactory fidelity, with no missing components or significant deviations. Anxiety decreased for all in the novel arm and half of the control. Stress decreased and coping increased for half in both arms. All participants increased and sustained BTA use and half with mindfulness activities. There were no reported study harms.

Conclusions: Although the combination of BTA and MBSR proved equal or slightly better to mutual aid therapy on all outcomes, recruitment of people with a formal HAND diagnosis from a single site was challenging. We recommend that future exploration of these techniques broaden to those aging with HIV with cognitive challenges regardless of a formal HAND diagnosis.

Trial registration: clinicaltrials.gov, NCT03483740. Registered 30 March 2018, https://clinicaltrials.gov/ct2/show/NCT03483740

\section{Background}

Aging with HIV has become a priority area of focus as the epidemic enters its fifth decade. In some countries, such as Canada and the United States, approximately half of the population living with HIV is now over fifty ${ }^{1,2}$. Longevity for people living with HIV, which now is comparable to the general population, is due to early initiation of and consistent adherence to combination antiretroviral therapy (cART) ${ }^{3,4}$. However, aging comorbidities may present at an earlier age and in greater frequency amongst HIV- 
positive people compared to their seronegative peers ${ }^{4,5,6}$. Amongst these comorbidities (e.g., frailty, depression, cancer), HIV-related cognitive impairment's prevalence of approximately $50 \%$ is among the most common 7,8 . This may be a result of HIV entering the nervous system in a relatively short timespan; in one study of 96 HIV-positive people, HIV entered the nervous system at a median 77 days following infection ${ }^{9}$. With the delay between transmission and diagnosis, the brain is exposed to potential impairment as a result of HIV replication and damage before treatment can adequately suppress the virus $^{10}$.

Cognitive impairment in people living with HIV has been predominantly classified as HIV-associated neurocognitive disorder (HAND) ${ }^{10}$. HAND is thought to result from structural damage to fronto-striatialthalamatory circuits in the brain, where viral penetration prevents these neural pathways from effectively mediating cognitive, motor, and behavioural function ${ }^{7-14}$. Severe forms of HAND have sharply decreased in the era of modern CART, however the milder forms persist and may be increasing in prevalence as the HIV-positive population ages into their geriatric years ${ }^{12}$. A longitudinal study of 1,306 men living with HIV in the United States found a direct link between changes in cognition and changes in health-related quality of life, as HIV-related cognitive impairment can be a significant source of stress and anxiety and can be difficult to cope with on a daily basis ${ }^{15}$. Biomedical interventions have as yet been unsuccessful in preventing or reversing the prevalence and severity of mild-to-moderate HAND ${ }^{10,16}$. In the absence of and even alongside an eventual pharmacological remedy, psychosocial approaches are needed to improve coping with HAND's symptoms ${ }^{17}$. Although existing research has illuminated unique cognitive challenges amongst people aging with $\mathrm{HIV}^{18}$, such as a higher prevalence of cognitive impairment at an earlier age ${ }^{19}$ than the general population and dual stigma associated with HIV and cognitive challenges ${ }^{20}$, psychosocial interventions have not yet been well tested for people aging with HIV and the optimal approach is unknown ${ }^{21}$.

A scoping review of non-pharmacological dementia interventions for people with mild cognitive impairment found that most interventions focused on cognitive training (i.e., Brain Training Activities, or BTA $)^{22}$. BTA is comprised of games and activities that can be delivered via computer, tablet, or with pen and paper $^{23}$. These activities focus on a specific cognitive domain (such as speed of processing) or cognition more broadly, based on an assessment of each individual's deficits ${ }^{23-25}$. The activities, when practised for 1-3 hours per week for 6-10 weeks, have been shown to improve self-reported outcomes of coping and ability in the cognitive domains that the games are targeted towards ${ }^{24,25}$.

Psychosocial interventions have also been trialed for people with mild-to-moderate dementia, with mindfulness approaches among the most frequently used modalities ${ }^{26}$. Group therapies are particularly recommended due to their inherent ability to foster social connection ${ }^{27}$. Mindfulness-Based Stress Reduction (MBSR) groups may be the preferential mindfulness approach for cognitive impairment due to its ability to reduce anxiety and cultivate calmness ${ }^{28}$. The MBSR curriculum of eight weekly $1.5-2$ hour 
sessions, which teaches meditation, breathing, and grounding techniques ${ }^{29}$, has been found to create lasting practices for emotional regulation in participants following the intervention's conclusion ${ }^{30}$.

Combination approaches, blending intervention models, may be better suited to the complexity of HIVassociated health problems than a single technique approach ${ }^{31}$. Given BTA's and MBSR's promising results with the general population living with dementia, this study combined these interventions into cognitive remediation group therapy (CRGT). CRGT was tested against mutual aid group therapy, widely considered the most recognizable form of group support and widely practiced in HIV and dementia ${ }^{27,31}$. These groups use the single technique of talk therapy whereby one or two facilitators - often social workers - help the group maintain respect, stay on topic, and explicate connection and shared experience between group members ${ }^{27}$. CRGT was tested against mutual aid in a pilot randomized, controlled trial for people aging with HAND to assess feasibility, acceptability, intervention fidelity, and exploratory outcomes.

\section{Objective}

This pilot trial's objective was to assess and compare the feasibility, acceptability, intervention fidelity, and exploratory outcomes (stress, anxiety, coping, use of mindfulness and brain training activities) of the novel and control arms, to determine if and how a study of this nature could be expanded in a larger trial.

\section{Methods}

\section{Trial design}

This was a pilot, parallel design, two-arm RCT that recruited people aging with HIV who had been diagnosed with mild-to-moderate HAND from a neurobehavioural research centre at a tertiary care hospital in Toronto, Canada. This trial was designed using a refinement framework ${ }^{32}$ to assess whether a trial of this nature could be upscaled and, if so, what the key considerations (i.e., participants, design, interventions, outcomes) would be for that larger study. Community-based participatory research guided this trial's design as a team of ten people aging with HIV and concerned about HAND were consulted in intervention selection, questionnaire design, and eligibility and recruitment considerations. Further, people aging with HIV were staff facilitators on both intervention arms. The study protocol has been published elsewhere ${ }^{33}$. No changes were made from protocol to completion.

\section{Participants}

People living with HIV were eligible to participate in this study if they were at least forty years of age, had a documented diagnosis of mild-to-moderate HAND from the recruiting neurobehavioural clinic, had last clinic visit after 1 January 2016, had been living with HIV for five years or more, consented to be contacted by the recruiting clinic for further research, and could commit to eight weekly sessions of group therapy in downtown Toronto. Participants were excluded from participation if their HAND diagnosis was asymptomatic or severe, if they had been admitted to a hospital in the past month, if they were unable to 
communicate in English, if they were unable to use a tablet for brain training activities, or if the research coordinator assessed that they would be disruptive in group therapy (e.g., if they made discriminatory comments). These criteria were set to precipitate a comfortable group atmosphere where participants may share a similar level of cognitive impairment while having progressed past the initial concerns of a recent HIV diagnosis.

\section{Interventions}

The novel and control arms both contained a form of group therapy that was comprised of a 3-hour orientation session and eight 3-hour weekly group sessions in downtown Toronto, Canada.

Cognitive remediation group therapy_(novel arm).

The novel arm of cognitive remediation group therapy (CRGT) was held at the Centre for Mindfulness Studies in Toronto and blended mindfulness-based stress reduction (MBSR) and brain training activities (BTA). Each weekly session was divided as two hours of manualized MBSR facilitated by a physician and a social worker followed by one hour of BTA facilitated by a peer aging with HIV. For the BTA component, participants were provided with access to a Samsung tablet and a 1-year license to BrainHQ training by PositScience. As BTA has predominantly been administered as a passive, individual exercise where a participant is given the software and written instructions for use with minimal follow-up ${ }^{25}$, a participatory approach was required to incorporate BTA into active group therapy. The BTA facilitator sought participant feedback on how to structure this component of CRGT; the decision was to have forty minutes of group discussion on training progress and challenges with twenty minutes dedicated to individual practice and troubleshooting of software features.

\section{Mutual aid group therapy_(control arm).}

The control arm of mutual aid group therapy was held at the AIDS Committee of Toronto and was facilitated by a social worker and a peer aging with HIV. The facilitators followed the intervention's manual ${ }^{33}$ of maintaining safety in the group while encouraging participants to share their experiences living with HIV-related cognitive impairment and associated coping strategies with one another. Facilitators of this model attempt to create connections between participants by identifying commonalities in their experiences with the aim of creating a community of people within the group that can support one another through shared challenges.

\section{Outcomes}

Feasibility was assessed against a priori targets that the research team agreed would be necessary to proceed with a larger trial, based on relevant literature ${ }^{34,35}$ and the team's experience with piloting and implementing interventions of this nature ${ }^{36,37}$. The targets, based on a sampling frame of forty people, were: $30 \%$ of those contacted agreed to participate, $25 \%$ completed the initial assessment, $20 \%$ attended 
the first orientation session of their treatment arm, $16 \%$ completed their entire arm's group series the final 3-month follow-up assessment.

Acceptability was measured via a satisfaction questionnaire conducted at the midpoint and endpoint of each arm's group therapy series. The questionnaire consisted of the Likert-style Helping Characteristics of Self-Help and Support Groups Measure ${ }^{38}(22$-items, $a=0.87)$ and open-ended questions about session length, number of sessions, activities used, and evaluation of facilitators that the researchers have used in previous pilot intervention studies.

For intervention fidelity, facilitators of both trial arms submitted weekly session reports that were comprised of checklists of therapy components and open-ended questions about activities completed in each group, dynamics between participants, and challenges of facilitation. An a priori target of a minimum of $80 \%$ of checklist therapy components per session was set, based on prior fidelity research ${ }^{39}$.

Exploratory outcomes of stress, anxiety, coping, use of mindfulness strategies, and use of brain training activities were assessed at three times: preintervention, postintervention, and 3-month follow-up. Standardized measures used were: HIV/AIDS Stress Scale ${ }^{40}(29$-items, Likert, $a=0.91)$, Anxiety in Cognitive Impairment and Dementia Scale ${ }^{41}$ (26-items, dyadic, $a=0.87$ ), Coping Self-Efficacy Scale of Health Problems ${ }^{42}$ (10-items, Likert, $a=0.85$, and the Five Facet Mindfulness Questionnaire-Short Form ${ }^{43}$ (24-items, $a=0.80$ ).

\section{Sample size}

Twelve to sixteen participants was chosen as the numbers necessary to initiate this pilot RCT as: a) that would permit six to eight people in each trial arm, which is ideal for group therapy of this nature ${ }^{44}$; and b) similar numbers have been used to assess other pilot group therapy studies 31,37 .

\section{Randomization}

A research coordinator enrolled participants by confirming their eligibility, attaining their informed consent, and collecting their availability for group therapy. Concealed allocation was then employed, whereby the first author provided the study sponsor with unique identifiers for each enrolled person and the sponsor then randomized participants in a 1:1 fashion using size two blocks to either the novel or control arm. These results were then communicated individually to each participant, alongside details regarding the schedule of their group therapy series.

\section{Blinding}

Intervention facilitators were blind to outcome assessments, which were conducted by a research coordinator. Participants in one arm were blind to participants in the other arm. Analysts were blind to participant identities. Participants were not blinded regarding the content of each arm, as the research coordinator discussed the two arms that participants could be assigned to when attaining their informed 
consent. The similarity of interventions - that they were both group therapies with one more focused on discussion (control) and the other on mindfulness and brain training (novel) - was described.

\section{Data Analysis}

For feasibility, proportions of participant recruitment, enrolment, and completion were calculated. Acceptability was analyzed through a spaghetti plot showing individual results of the satisfaction measure and reasons for participant withdrawal were also described. Fidelity was analyzed by tabulating intervention checklist items and qualitatively by two independent coders trained in the intervention models who employed content analysis for the open-ended responses to determine if interventions were delivered as designed. Exploratory outcomes were also analyzed through spaghetti plots show individual progress on these assessments, complemented by non-parametric Wilcoxon rank sum tests run in R 3.5.1 (Feather Spray) to determine if there was a significant between group difference for change in outcomes relative to preintervention. As the team could not find a standardized scale to assess BTA use at the time of study design, progress reports from PositScience by BrainHQ were used for the novel arm and selfreported use of BTA was used for the control arm.

\section{Results}

The participant flow and demographics are listed below, followed by outcome results. Quotes are from facilitator session reports and specify the intervention arm and session number.

\section{Participant flow}

Figure 1 displays participant flow throughout the study. There were one hundred individuals diagnosed with HAND at the recruiting clinic since 1 January 2016, of which forty were diagnosed with the mild-tomoderate form that comprised the study's sampling frame.

\section{Recruitment}

From 1 April to 30 September 2018, a clinical psychologist from the recruiting site made three distinct contact attempts to all participants in the sampling frame $(n=40)$ at the phone number and email address that they provided at their last clinic visit. These contacts briefly detailed the study and eligibility criteria, and asked participants whether they would like to be contacted by the research coordinator to schedule a meeting to confirm eligibility and review the consent form. Twelve people consented to participate, on the lower end of our target sample. Since the sampling frame's contact attempts had been exhausted and some people had been waiting approximately four months for the trial to begin, the trial was initiated with twelve participants on 1 October 2018 with the study sponsor generating the random assignment. Both groups were held on Wednesdays, with the novel arm in the afternoon and the control arm in the evening. Preintervention outcome assessments were conducted on 10 October 2018. Group series ran from 17 October 2018 to 12 December 2018. Postintervention outcomes were assessed on 19 December 2018 
and the 3-month follow-up was conducted on 19 March 2018. Participants received \$20 Canadian honourarium for each study visit, and light refreshments were provided for each group session.

\section{Baseline data}

Participants ( $\mathrm{n}=12)$ all identified as gay, cisgender males who had been diagnosed with mild-to-moderate HAND. All participants also had a family doctor and an HIV specialist, were taking daily antiretroviral HIV medication, and identified prescription substance misuse. Refer to table 1 for further participant characteristics.

\begin{tabular}{|c|c|c|}
\hline & Novel Arm n/Mean & Control Arm n/Mean \\
\hline Age & 51.5 & 59.7 \\
\hline Race & & \\
\hline Caucasian & 5 & 4 \\
\hline Afrocaribbean/Black & 1 & 0 \\
\hline Hispanic & 0 & 2 \\
\hline Income source & & \\
\hline Employment & 2 & 1 \\
\hline Disability support & 4 & 4 \\
\hline Other (Pension, CPP) & 0 & 1 \\
\hline Number of years living with HIV & 19.3 & 19.33 \\
\hline CD4 at most recent visit & 608 & 597 \\
\hline Total number of non-HIV prescription medications & 6.3 & 4 \\
\hline Current Substance Use & & \\
\hline Alcohol & 4 & 3 \\
\hline Tobacco & 1 & 1 \\
\hline Prescription substance misuse & 6 & 6 \\
\hline Other non-prescribed substance use & 3 & 4 \\
\hline Current Axis I Diagnoses (Lifetime) & & \\
\hline Substance Related Disorder & 1 & 2 \\
\hline Psychotic disorder (schizophrenia; schizoaffective disorder) & 2 & 3 \\
\hline Mood disorder (MDD; bipolar disorder) & 6 & 5 \\
\hline
\end{tabular}

\section{Numbers analyzed}

Feasibility results are presented against a denominator of forty, which is the number of people that the recruiting psychologist attempted to contact. For all other outcomes, the denominator is ten, the number of participants who completed the study and all assessments.

\section{Outcomes}

The trial met its a priori targets for feasibility and fidelity, and the novel arm's CRGT model proved equal or slightly better than the control's mutual aid model on acceptability and all exploratory outcomes. The 
fidelity measures also offered qualitative insight into the nuances of facilitating these interventions for people aging with HIV and cognitive challenges.

Feasibility

Overall, $30 \%(n=12)$ of the sampling frame agreed to participate, completed the preintervention assessment, and attended the first orientation session of their arm's intervention. Two participants (one from each arm) withdrew from the trial during the interventions, leaving $25 \%(n=10)$ of the sampling frame that completed their arm's group series and the final 3-month follow-up study visit.

Acceptability.

On the standardized satisfaction scale at postintervention, the novel arm averaged $90 \%$ acceptability compared to $85 \%$ in the control. Individual plots of acceptability scores can be found in Figure 2. Openended acceptability responses were brief but positive in both arms, as participants stated that they felt session and series length were appropriate, the facilitators were skilled and non-judgmental, and that the groups' activities were helpful.

Fidelity

Facilitators self-reported use of intervention components via checklists, and added additional qualitative detail to support their claims. Two independent coders found that both arms were delivered with satisfactory fidelity. Details on each intervention component are provided below.

\section{Mindfulness-based stress reduction (MBSR)}

The first component of the novel CRGT arm was mindfulness-based stress reduction (MBSR), which follows a manual that closely details activities including body scans, meditations, and deep breathing exercises. Each week's prescribed MBSR components were endorsed by facilitators. An illustrative quote of MBSR in practice in this study is:

Participants identified body scans as making a big difference. They stated that they have felt so heavy since receiving a HAND diagnosis and feeling unable to share this experience with others due to fears of stigma. By focusing attention on their bodies in the present, they shift away from negative thoughts about perceived discrimination and say they feel less anxious and agitated. (CRGT novel arm, session 7)

\section{Brain training activities (BTA)}

The second component of the novel arm involved a peer facilitator leading discussion on progress and challenges with tablet-based BTA, and time dedicated to practice with the activities. The facilitator stated that all sessions sustained this plan, and that participants stated that, through repeated practice, speedof-processing and memory recall events in their daily lives appeared to become easier. The facilitator identified that the group setting was facilitative of BTA practice: 
The group had frequent conversations about navigating feelings of frustration when they don't perform well on a brain training activity. Members shared strategies such as taking a break, switching to a brain training activity that felt easier for a while to bolster confidence, and taking deep breaths and doing a body scan. Members stated that group discussion of brain training activities kept them motivated to continue practicing. (CRGT novel arm, session 5)

\section{Mutual aid group therapy}

Mutual aid involves a structured check-in and check-out process to each session, with facilitators providing consistent therapeutic skills such as active listening, reflecting feelings, and presenting similarities in participant experiences. These components were endorsed by facilitators and confirmed by the independent assessors. A quote from facilitator reports that sums up this group's experience is as follows:

Group members marvel at the new experience of being able to discuss cognitive challenges and HIV with others experiencing similar issues, as they have never felt comfortable opening up about these topics before. Members commented on the commonalities they have found in the group, and how much they have learned from each other about coping with HAND. (Mutual aid control arm, session 8)

\section{Exploratory outcomes}

Figure 3 displays individual results of exploratory outcome assessments of anxiety, stress, coping, and use of mindfulness activities at preintervention (T1), postintervention (T2), and 3-month follow-up (T3). Anxiety decreased for all participants in the CRGT novel arm and for half of the mutual aid control, although a change was not significantly different between the two groups at T3 relative to T1 $(p=0.17)$. Stress decreased for approximately half of the participants in each arm, and this decrease was significantly greater in the novel arm compared to the control at T3 relative to T1 $(p<0.01)$. Coping also improved for about half of each group, with an insignificant difference between the novel arm and control at T3 compared to T1 $(p=0.75)$. Half of each group's participants increased in use of mindfulness activities with the novel arm's increase being significantly greater than the control's at T3 compared to T1 $(p=0.035)$. Based on usage data from PositScience by BrainHQ, the novel arm's six participants sustained over three hours of BTA practice per week for the 21-week duration of the study. The control arm's six participants self-reported BTA use of 1-3 hours per week during the study's timeline.

\section{Harms}

There were no reported study-related harms.

\section{Discussion}

The principal findings of this pilot are that group therapy may be feasible and acceptable to people aging with HIV and cognitive challenges and, further, that a complex intervention combining mindfulness and brain training activities may be preferential to the standard mutual aid type of group support. Compared 
with other studies, a strength of this pilot was its use of an active comparator as mindfulness trials are often limited by an inactive control ${ }^{45}$. Another strength was the study's involvement of people aging with HIV and community-based organizations as this community connection may enhance the pilot's potential for a full-scale trial following refinement ${ }^{32}$.

Compared with other studies, the consistent practice of BTA and MBSR activities was unusually sustained for a three-month follow-up. This may be attributable to the CRGT model of administering these interventions together, in a group setting. BTA has been delivered as a predominantly individual intervention ${ }^{25}$, whereby brain training software or activity books are given to people individually with the expectation that they will practice in isolation ${ }^{46}$. This passive approach leads to high drop-off rates; while completers receive the self-reported coping benefit discussed above, upwards of half of a BTA trial's participants do not adhere to the training regimen as prescribed ${ }^{23-25}$. MBSR has stronger completion rates, possibly due to its delivery in group settings where participants attend not solely for their own therapy but also for the social connection inherent in support groups ${ }^{30}$. Yet, MBSR participants living with mild-to-moderate dementia bemoan the intervention's lack of practical resources to address their cognitive deficits $^{28}$. Therefore, the novel CRGT arm blending the practical (e.g., BTA) and emotional (e.g., MBSR) may offer an intervention package that supports regular practice of these activities, enhancing their potential to ameliorate the effects of cognitive impairment.

\section{Limitations}

This trial has several limitations. A small sample size limits outcome assessment and interpretation. This small sample may be the result of limiting recruitment to a single site and/or a formal diagnosis of mildto-moderate HAND, which requires intensive neuropsychological screening. The lack of participant blinding poses a risk to performance bias whereby participant responses may have been affected by allocation to the novel or control arm. Assessing fidelity from facilitator reports risks reporting bias, where the facilitators may have exaggerated the groups' progress. Recording sessions may be a more rigourous measure of fidelity. With MBSR and BTA both containing non-verbal exercises, video recording would be preferential to audio although this population's concerns regarding stigma and privacy related may make them unduly uncomfortable with recorded sessions.

\section{Applicability}

Group therapy for people aging with HIV and cognitive challenges is a novel form of support for this population, and may ease the burden of living with these comorbid challenges. This pilot found that a complex group intervention that involved physical movement of MBSR, talk therapy, and online BTA use with a tablet were all feasible and acceptable intervention components for participants. Requiring a formal HAND diagnosis may limit eligibility too greatly, given the intensive screening requirements and the lack of sites that offer screening. Broadening these intervention techniques to people aging with HIV with cognitive challenges regardless of a formal HAND diagnosis may enhance the success of a larger study. 


\section{Interpretation}

While the small sample requires that this trial's positive results be interpreted with some caution, we found no evidence of study-related harms. As MBSR and BTA have helped ameliorate the stress and anxiety of cognitive decline in the general population with dementia, and given that their combination here was feasible and acceptable to people aging with HIV, the continuation of this expansion of this CRGT approach may be justified for this population. A larger trial would require a more substantial sampling frame, likely consisting of multiple sites. To feasibly implement a larger trial of this nature with similar community connectedness, a pragmatic trial design such as a stepped wedge waitlist crossover may be the ideal method since it would offer CRGT to all participants, eventually, while assessing outcomes against a waitlisted control. Since this study was designed, measures such as the HIV Disability Questionnaire ${ }^{47}$ and the Rotterdam Healthy Aging Score ${ }^{48}$ have emerged that may be more suitable to outcome assessments in this population.

\section{Conclusion}

This pilot study presents results from the first known implementation of group therapy for people with HAND, with a multifaceted evaluation and an active comparator. A larger trial is needed to determine the effectiveness of group therapy for people aging with HIV and cognitive challenges.

\section{Abbreviations}

AIDS

Acquired Immunodeficiency Syndrome

BTA

Brain Training Activities

CRGT

Cognitive Remediation Group Therapy

HAND

HIV-Associated Neurocognitive Disorder

HIV

Human Immunodeficiency Virus

MBSR

Mindfulness-Based Stress Reduction

\section{Declarations}

\section{Ethics approval and consent to participate}

This study was approved by the Research Ethics Boards of St. Michael's Hospital (17-334) and the University of Toronto (35860). Informed consent was obtained from all study participants. 


\section{Consent for publication}

Not applicable.

\section{Availability of data and materials}

The data used and analyzed during the current study are available from the corresponding author on reasonable request.

\section{Competing interests}

The authors declare that they have no competing interests.

\section{Funding}

This study was funded by the CIHR Canadian HIV Trials Network (CTN) through a Pilot Study Grant (PT029). ADE's salary is supported by the Ontario HIV Treatment Network (OHTN) through a Student Leader Award in HIV and Aging. SLC and BAF are Canada Research Chairs. SLW receives salary support from the OHTN through a Research Chair Award in HIV Clinical Care and Aging. The funders did not have a role in the study's design, nor in the decision to submit the study for publication.

\section{Authors' contributions}

ADE oversaw study activities, supervised personnel, and wrote the first manuscript draft. SLC provided expertise with intervention design. SBR and TS provided access to their clinic's population and recruited participants. BAF and JWM contributed to protocol refinement. SLW conceptualized the study with ADE and provided expertise in trial design. All authors edited and approved the final manuscript.

\section{Acknowledgements}

We wish to thank all our study participants for their voluntary participation, and all of the people aging with HIV who contributed to the study's design, implementation, and dissemination. Thanks to Dr. Judy Needham, Erica Jaaf, and Dr. Terry Lee at the CIHR Canadian HIV Trials Network (CTN) for excellent project management, data management, and statistical consultation, respectively. Thanks to Bob Wallace, Dr. Patricia Rockman, Gwen Morgan, Constantine Cabarios, and Mike Slonimsky for facilitating the trial's interventions. Thanks to Jenny Hui and Nelson Pang for assistance with this article.

\section{Registration}

Trial registration: clinicaltrials.gov, NCT03483740. Registered 30 March 2018, https://clinicaltrials.gov/ct2/show/NCT03483740

\section{Protocol}


The study protocol has been published in $B M J$ Open and is available here: https://bmjopen.bmj.com/content/9/10/e033183

\section{References}

1. Bourgeois AC, Edmunds M, Awan A, Jonah L, Varsaneux O, Siu W. HIV in Canada - surveillance report. Can Commun Dis Rep. 2017;43(12):248-256. doi:10.14745/ccdr.v43i12a01

2. HIV among people aged 50 and older. Centres for Disease Control and Prevention. 2018. https://www.cdc.gov/hiv/group/age/olderamericans/index.html

3. Wandeler G, Johnson LF, Egger M. Trends in life expectancy of HIV-positive adults on ART across the glove: comparisons with general population. Curr Opin HIV AIDS. 2016;11(5):492-500.

doi: $10.1097 / \mathrm{COH} .0000000000000298$

4. Marcus JL, Chao CR, Leyden WA, Xu L, Quesenberry CP Jr, Klein DB, et al. Narrowing the gap in life expectancy between HIV-infected and HIV-uninfected individuals with access to care. J Acquir Immune Defic Syndr. 2016;73(1):39-46. doi:10.1097/QAI.0000000000001014

5. Ball SC. Increased longevity in HIV: caring for older HIV-infected adults. Care Manag J. 2014;15(2);76-82. doi:10.1891/1521-0987.15.2.76

6. Maciel RA, Klück HM, Durand M, Sprinz E. Comorbidity is more common and occurs earlier in persons living with HIV than in HIV-uninfected matched controls, aged 50 years and older: a crosssectional study. Int J Infec Dis. 2018;70:30-5. doi:10.1016/j.ijid.2018.02.009

7. Greene M, Covinsky KE, Valcour V, Miao Y, Madamba J, Lampiris H, et al. Geriatric syndromes in older HIV-infected adults. J Acquir Immune Defic Syndr. 2015;69(2):161-7. doi:10.1097/QAI.0000000000000556

8. Valcour V. HIV, aging, and cognition: emerging issues. Top Antivir Med. 2013;21(3):119-23. PMID:23981600

9. Spudich S. HIV and neurocognitive dysfunction. Curr HIV/AIDS Rep. 2013;10:235-243. doi:10.1007/s11904-013-0171-y

10. Antinori A, Arendt G, Grant I, Letendre S, Muñoz-Moreno JA, Eggers C, et al. Assessment, diagnosis, and treatment of HIV-associated neurocognitive disorder: a consensus report of the mind exchange program. Clin Infect Dis 2013;56:1004-17. doi:10.1093/cid/cis975

11. Grant I, Franklin DR, Deutsch R, Woods SP, Vaida F, Ellis RJ, et al. Asymptomatic HIV-associated neurocognitive impairment increases risk for symptomatic decline. Neurology. 2014;82:205562. doi:10.1212/WNL.0000000000000492

12. Heaton RK, Clifford DB, Franklin DR, Woods SP, Ake C, Vaida F, et al. HIV-associated neurocognitive disorders persist in the era of potent antiretroviral therapy: charter study. Neurology. 2010;75:208796. doi:10.1212/WNL.0b013e318200d727

13. Antinori A, Arendt G, Becker JT, Brew BJ, Byrd DA, Cherner M, et al. Updated research nosology for HIV-associated neurocognitive disorders. Neurology. 2007;69:1789-99. 
doi:10.1212/01.WNL.0000287431.88658.8b

14. Clifford DB, Ances, BM. HIV-associated neurocognitive disorder (HAND). Lancet Infect Dis 2013;13:976-86. doi:10.1016/S1473-3099(13)70269-X

15. Jones JD, Kuhn T, Levine A, Sacktor N, Munro CA, Teplin LA, et al. Changes in cognition precede changes in HRQoL among HIV+ males: longitudinal analysis of the multicenter AIDS cohort study. Neuropsychol. 2019;33(3):370-8. doi:10.1037/neu0000530

16. Al-Khindi T, Zakzanis KK, van Gorp WG. Does antiretroviral therapy improve HIV-associated cognitive impairment? a quantitative review. J Int Neuropsychol Soc. 2011;17:956-9. doi:

$10.1017 /$ S1355617711000968

17. Tedaldi E, Minniti N, Fischer T. HIV-associated neurocognitive disorders: the relationship of HIV infection with physical and social comorbidities. BioMed Res Int. 2015;641913. doi:10.1155/2015/641913

18. Eaton AD, Craig SL, Wallace R. The intersecting cognitive and aging needs of HIV-positive older adults: implications for social work practice. Soc Work Health Care 2017;56:733-47. doi:10.1080/00981389.2017.1339759

19. Milanini B, Valcour V. Differentiating HIV-associated neurocognitive disorders from Alzheimer's disease: an emerging issue in geriatric neuro HIV. Curr HIV/AIDS Rep 2017;14;123-132. doi: 10.1007/s11904-017-0361-0

20. Hopcroft L, Bester L, Clement D, Quigley A, Sachdeva M, Rourke SB, et al. "My body's a 50 year-old but my brain is definitely an 85 year-old: exploring the experiences of men ageing with HIVassociated neurocognitive challenges. J Int AID Soc 2013;16;18506. doi:10.7448/IAS.16.1.18506

21. Illa L, Echenique M, Bustamante-Avellaneda V, Sanchez-Martinez M. Review of recent behavioral interventions targeting older adults living with HIV/AIDS. Curr HIV/AIDS Rep 2014;11;413-22. doi:10.1007/s11904-014-0231-y

22. Rodakowski J, Saghafi E, Butters MA, Skidmore ER. Non-pharmacological interventions for adults with mild cognitive impairment and early stage dementia: an updated scoping review. Mol Aspects Med 2015;43;38-53. doi:10.1016/j.mam.2015.06.003

23. Barnes DE, Yaffe K, Belfor N, Jagust WJ, DeCarli C, Reed, BR, et al. Computer-based cognitive training for mild cognitive impairment: results from a pilot randomized, controlled trial. Alzheimer Dis Assoc Disord 2009;23;205-10. doi:10.1097/WAD.0b013e31819c6137

24. Boripuntakul S, Kothan S, Methapatara P, Munkhetvit P, Sungkarat S. Short-term effects of cognitive training program for individuals with amnestic mild cognitive impairment: a pilot study. Phys Occup Ther Geriatr 2012;30;138-149. doi:0.3109/02703181.2012.657822

25. Tappen RM, Hain D. The effect of in-home cognitive training on functional performance of individuals with mild cognitive impairment and early-stage Alzheimer's disease. Res Gerontol Nurs 2014;7;14-24. doi:10.3928/19404921-20131009-01

26. Berk L, Warmenhoven F, van Os J, van Boxtel M. Mindfulness training for people with dementia and their caregivers: rationale, current research, and future directions. Front Psychol 2018;9;982. 
doi:10.3389/fpsyg.2018.00982

27. Toms GR, Clare L, Nixon J, Quinn C. A systematic narrative review of support groups for people with dementia. Inl Psychogeriatr 27;1439-65. doi:10.1017/S1041610215000691

28. Russell-Williams J, Jaroudi W, Perich T, Hoscheidt S, El Haj M, Moustafa AA. Mindfulness and meditation: treating cognitive impairment and reducing stress in dementia. Rev Neurosci 2018;29;791-804. doi:10.1515/revneuro-2017-0066

29. Kabat-Zinn J, Blacker M, Herbette G, Fulwiler C. Mindfulness-based stress reduction (MBSR) authorized curriculum guide. 2017. https://www.umassmed.edu/globalassets/center-formindfulness/documents/mbsr-curriculum-guide-2017.pdf

30. Goldin PR, Gross JJ. Effects of mindfulness-based stress reduction (MBSR) on emotion regulation in social anxiety disorder. Emotion 2010;10;83-91. doi:10.1037/a0018441

31. Bateganya MH, Amanyeiwe U, Roxo U, Dong M. Impact of support groups for people living with HIV on clinical outcomes: a systematic review of the literature. J Acquir Immune Defic Syndr 2015;68; S368-74. doi:10.1097/QAl.0000000000000519

32. Justice J, Miller JD, Newman JC, Hashmi SK, Halter J, Austad SN, et al. Frameworks for proof-ofconcept clinical trials of interventions that target fundamental aging processes. J Gerontol A Biol Sci Med Sci 2016;71;1415-23. doi:10.1093/gerona/glw126

33. Eaton AD, Walmsley SL, Craig SL, Rourke SB, Sota T, McCullagh JW, et al. Protocol for a pilot randomised controlled trial evaluating feasibility and acceptability of cognitive remediation group therapy compared with mutual aid group therapy for people ageing with HIV-associated neurocognitive disorder (HAND) in Toronto, Canada. BMJ Open 2019;9:e033183. doi:10.1136/bmjopen-2019-033183

34. Eldridge SM, Lancaster GA, Campbell MJ, Thabane L, Hopewell S, Coleman CL, et al. Defining feasibility and pilot studies in preparation for randomised controlled trials: Development of a conceptual framework. PLoS ONE 2016;11;e0150205. doi:10.1371/journal.pone.0150205

35. Thabane L, Ma J, Chu R, Cheng J, Ismaila A, Rios LP, et al. A tutorial on pilot studies: the what, why and how. BMC Med Res Methodol 2010;10. doi:10.1186/1471-2288-10-1

36. Eaton AD, Chan Carusone S, Craig SL, Telegdi E, McCullagh JW, McClure D, et al. The ART of conversation: feasibility and acceptability of a pilot peer intervention to help transition complex HIVpositive people from hospital to community. BMJ Open 2019;9;e026674. doi:10.1136/bmjopen-2018026674

37. Eaton AD, Cattaneo JS, Watchorn JM, Bilbao-Joseph C, Bowler S, Hazelton M, et al. From serodiscordant to magnetic: the feasibility and acceptability of a pilot psychoeducational group intervention designed to improve relationship quality. Soc Work Groups 2018;41;323-35. doi:10.1080/01609513.2017.1369922

38. Schiff M, Bargal D. Helping characteristics of self-help and support groups: their contribution to participants' subjective well-being. Small Group Res 2003;31;275-304. 
39. Borrelli B. The assessment, monitoring, and enhancement of treatment fidelity in public health clinical trials. J Public Health Dent 2011;71;S52-S63. doi:10.1111/j.1752-7325.2011.00233.x

40. Packenham KJ, Rinaldis M. HIV/AIDS stress scale. Psych \& Health 2003;17;203-19.

41. Gerolimatos LA, Ciliberti CM, Gregg JJ, Nazem S, Bamonti PM, Cavanagh CE, et al. Development and preliminary evaluation of the anxiety in cognitive impairment and dementia (ACID) scales. Int Psychogeriatrics 2015;27;1825-38. doi:10.1017/S1041610215001027

42. Gandoy-Crego M, Clemente M, Gómez-Cantorna C, González-Rodriguez R, Reig-Botella A. Selfefficacy and health: the SEH scale. Am J Health Behav 2016;40;389-95. doi:10.5993/AJHB.40.3.11

43. Bohlmeijer E, ten Klooster PM, Fledderus M, Veehof M, Baer R. Psychometric properties of the five facet mindfulness questionnaire in depressed adults and development of a short form. Assessment 2011;18;308-20. doi:10.1177/1073191111408231

44. Lenferink LIM, de Keijser J, Wessel I, Boelen PA. Cognitive behavioural therapy and mindfulness for relatives of missing persons: a pilot study. Pilot Feasibility Stud 2019;5;93. doi:10.1186/s40814-019$0472-z$

45. Dunning D, Griffiths K, Kuyken W, Crane C, Foulkes L, Parker J, et al. Research review: the effects of mindfulness-based interventions on cognition and mental health in children and adolescents - a meta-analysis of randomized controlled trials. J Child Psychol Psychiatry 2019;60;244-58. doi: $10.1111 /$ jcpp. 12980

46. Vance DE, Jensen M, Tende F, Raper JL, Morrison S, Fazeli PL. Individualized-targeted computerized cognitive training to treat HIV-associated neurocognitive disorder: an interim descriptive analysis. J Assoc Nurses AIDS Care 2018;29;604-11. doi:10.1016/j.jana.2018.04.005

47. Brown DA, Simmons B, Boffito M, Aubry R, Nwokolo N, Harding R, et al. Evaluation of the psychometric properties of the HIV disability questionnaire among adults living with HIV in the United Kingdom: a cross-sectional self-report measurement study. PLoS ONE 2019;14;e0213222. doi:10.1371/journal.pone.0213222

48. Walmsley SL, Ren M, Simon C, Clarke R, Szadkowski L. Pilot study assessing the Rotterdam healthy aging score in a cohort of human immunodeficiency virus positive adults in Toronto, Canada. AIDS 2020. doi:10.1097/QAD.0000000000002493

\section{Figures}




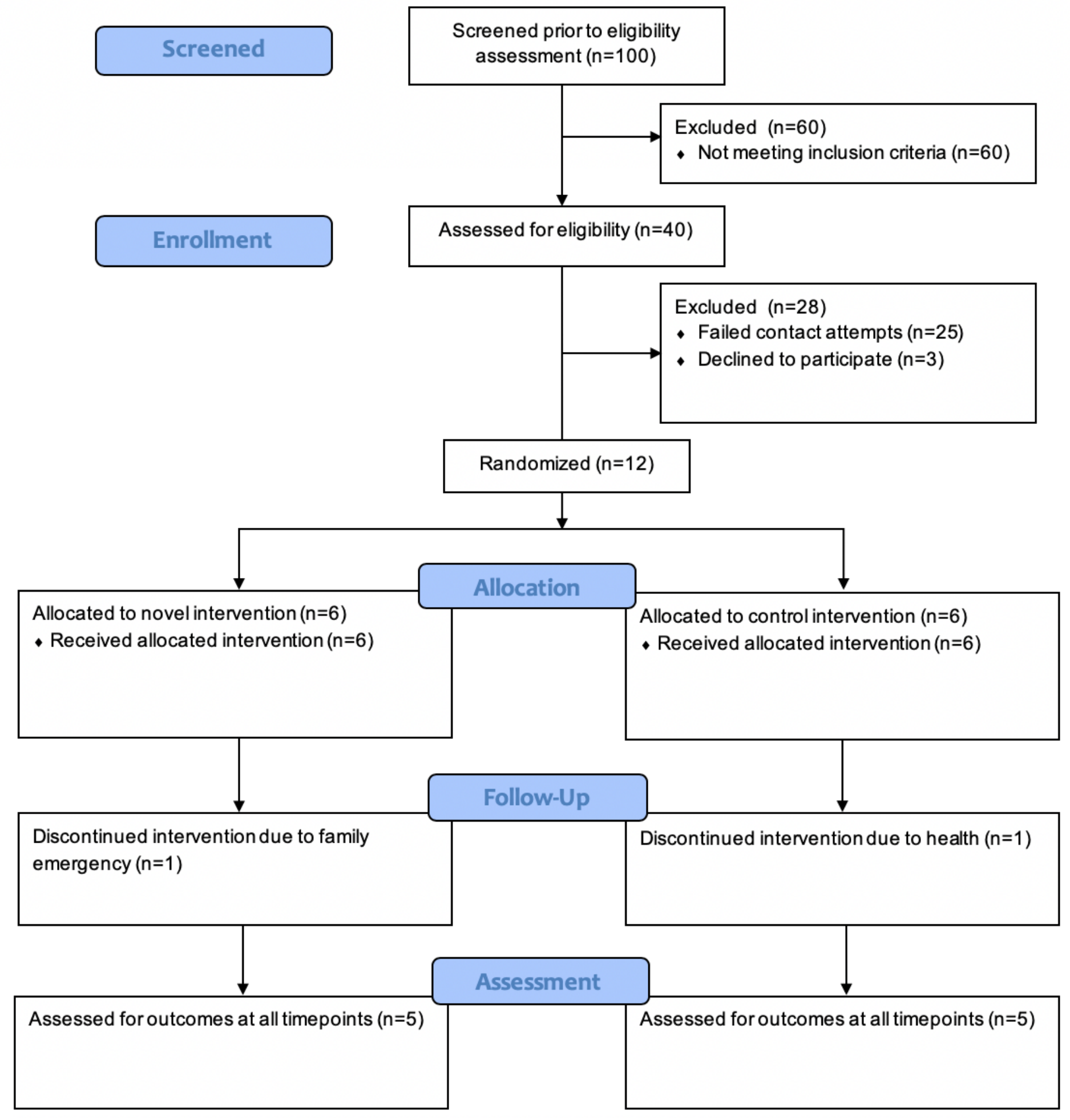

Figure 1

Participant flowchart 


\section{Acceptability}

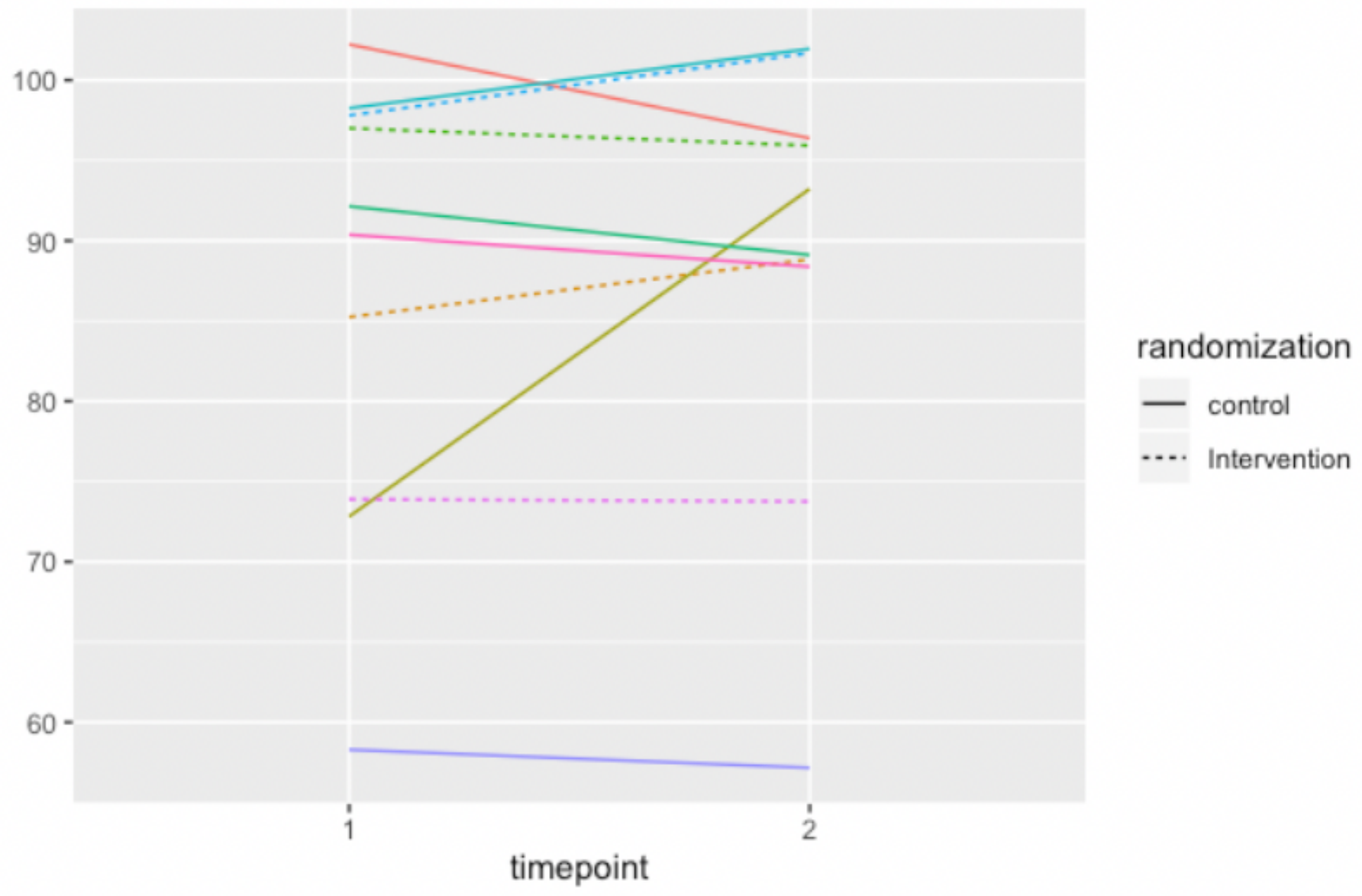

Figure 2

Acceptability scores at midintervention and postintervention 


\section{Anxiety}

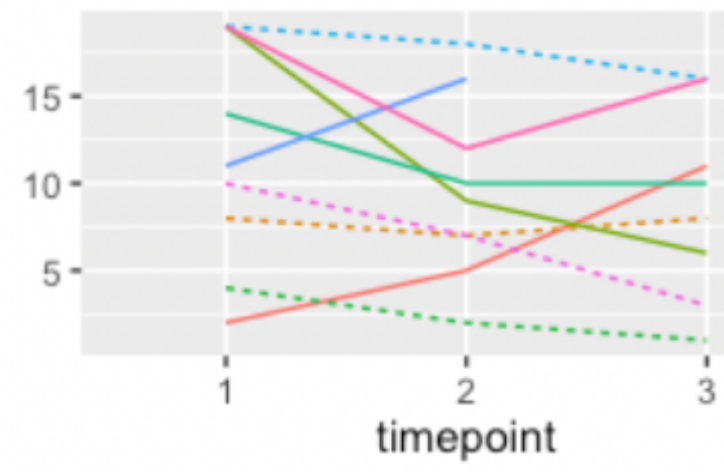

\section{Coping}

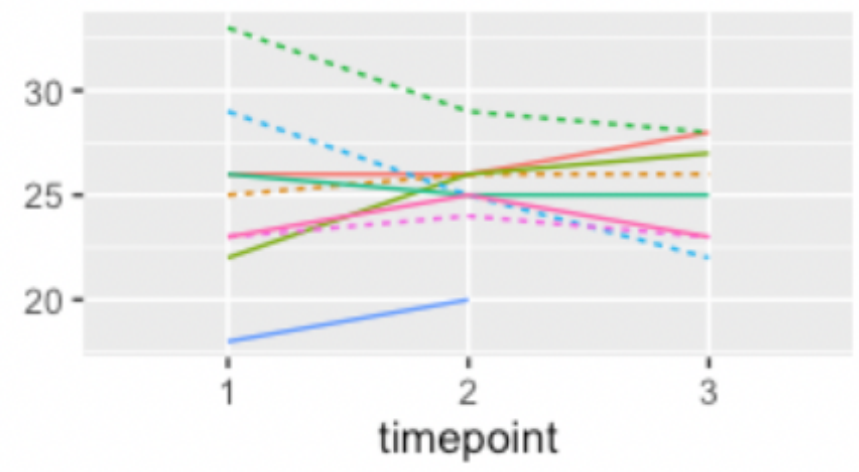

Stress

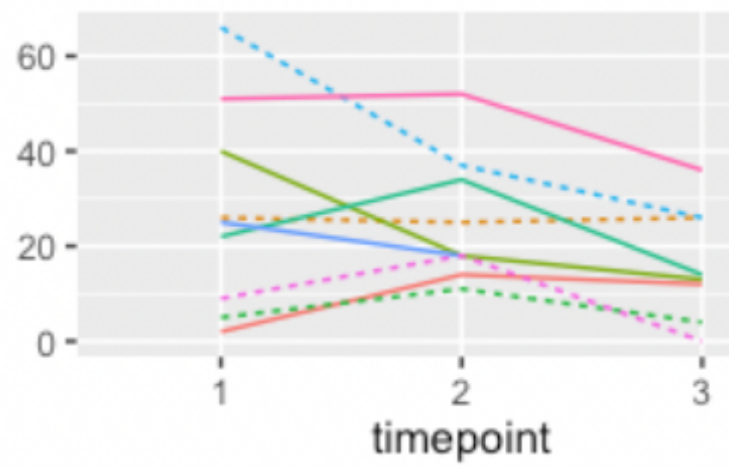

\section{Mindfulness}

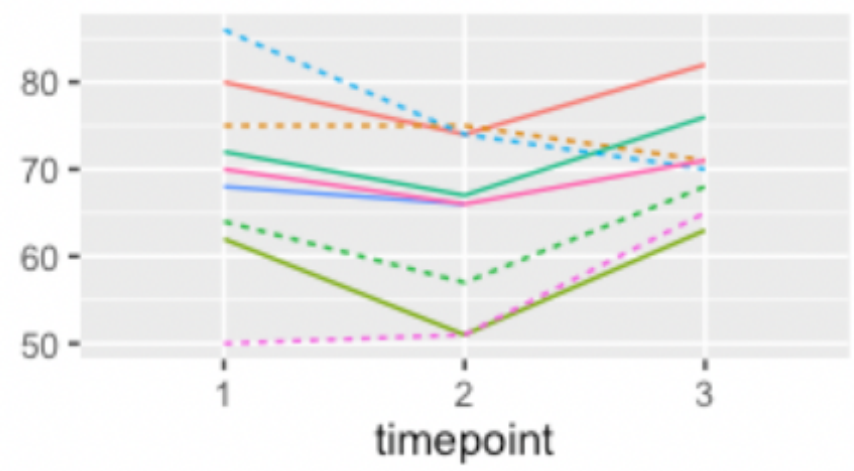

randomization - control $\cdots \cdot$ Intervention

\section{Figure 3}

Exploratory outcomes at preintervention, postintervention, and 3-month follow-up

\section{Supplementary Files}

This is a list of supplementary files associated with this preprint. Click to download.

- CONSORTextensionforPilotandFeasibilityTrialsChecklist.doc 